\title{
Exosomal IncRNA-ATB activates astrocytes that promote glioma cell invasion
}

\author{
ER-BAO BIAN ${ }^{1,2}$, ER-FENG CHEN $^{1,2}$, YA-DI XU ${ }^{1,2}$, ZHI-HAO YANG $^{1,2}$, \\ FENG TANG $^{1,2}$, CHUN-CHUN MA ${ }^{1,2}$, HONG-LIANG WANG ${ }^{1,2}$ and BING ZHAO ${ }^{1,2}$ \\ ${ }^{1}$ Department of Neurosurgery, The Second Affiliated Hospital of Anhui Medical University; \\ ${ }^{2}$ Cerebral Vascular Disease Research Center, Anhui Medical University, Hefei, Anhui 230601, P.R. China
}

Received July 16, 2018; Accepted October 29, 2018

DOI: $10.3892 /$ ijo.2018.4644

\begin{abstract}
Glioma invasion is a main cause of a poor prognosis and relapse in patients suffering from the disease. However, the molecular mechanisms responsible for glioma cell invasion remain poorly understood. In this study, the characteristics of exosomes were identified using electron microscope (TEM), and western blot analysis. The potential mechanism of long non-coding RNA (lncRNA) activated by TGF- $\beta$ (lncRNA-ATB) was demonstrated using luciferase reporter assays and RNA immunoprecipitation. We found that glioma cell-derived exosomes promoted the activation of astrocytes and had the ability to shuttle long non-coding RNA (lncRNA) activated by TGF- $\beta$ (lncRNA-ATB) to astrocytes. More importantly, IncRNA-ATB activated astrocytes through the suppression of microRNA (miRNA or miR)-204-3p in an Argonaute 2 (Ago2)-dependent manner. Furthermore, astrocytes activated by IncRNA-ATB in turn promoted the migration and invasion of glioma cells. Taken together, the findings of this study suggest that IncRNA-ATB may play an important role in modulating glioma microenvironment through exosomes. Thus, a better understanding of this process may provide implications for the prevention of highly invasive glioma.
\end{abstract}

\section{Introduction}

Despite the standard of care, consisting of surgical resection combined with chemotherapy and radiotherapy, the prognosis

Correspondence to: Professor Bing Zhao, Department of Neurosurgery, The Second Affiliated Hospital of Anhui Medical University, 678 Fu Rong Road, Hefei, Anhui 230601, P.R. China E-mail: aydbeb@126.com; aydzhb@126.com

Abbreviations: GBM, glioblastoma multiforme; EVs, extracellular vesicles; MVBs, multivesicular bodies; lncRNAs, long non-coding RNAs; lncRNA-ATB, long non-coding RNA activated by TGF- $\beta$; HCC, hepatocellular carcinoma; EMT, epithelial-mesenchymal transition; TEM, transmission electron microscopy; NHAs, normal human astrocytes; ceRNAs, competitive endogenous RNAs

Key words: glioma, astrocytes, long non-coding RNA-ATB, microRNA-204-3p of patients with glioma remains dismal (1). A number of factors promote the malignant potential of glioma, including the capacity of glioblastoma multiforme (GBM) to regulate immune responses both locally and systemically (2). Increasing evidence indicates that cancer cells can change the normal cell phenotype by the secretion of growth factors, chemokines and cytokines, which in turn promotes tumor growth, invasion and metastases $(3,4)$. In the brain, chronic inflammation due to the presence of glioma cells results in the infiltration of reactive astrocytes, which are major components of the invasive niche at the interface of glioma cells (5-7). Tumor-stroma interactions are in part mediated by secreting soluble factors, such as growth factors. However, the mechanisms underlying the communication between astrocytes and glioma cells are complex, and exosomes are likely involved.

Exosomes are small (30-100 $\mathrm{nm})$ vesicles secreted from cells into the extracellular space (8). Exosomes are formed by an inward budding of the plasma membrane in the late endosomes, thus enclosing within the internal vesicles to form multivesicular bodies (MVBs) within the cytoplasm, which may fuse with the plasma membrane and release its content of exosomes (9). Exosomes contain a set of specific proteins, including tetraspanins (CD9, CD63 and CD81), TSG101, Alix and flotillin (10). Exosomes are highly enriched in sphingomyelin, hexosylceramides and cholesterol at the expense of phosphatidylethanolamine and phosphatidylcholine (11). Several crucial signals, including small GTPases, the endosomal sorting complex required for transport (ESCRT) and the SNARE complex are involved in exosome release (12). Although they were initially considered as removal of the garbage for cells, more recently exosomes have been shown to mediate intercellular communications $(13,14)$. Exosomes are released by many cell types into the extracellular environment and are found in a variety of body fluids, such as saliva, urine, blood, ascites, breast milk and cerebrospinal fluid (15). Exosomes carry a specific payload of fully functional proteins, lipids and nucleic acids when transferred into recipient cells (16-18). Functionally, exosomes have been implicated in the modulation of host immune responses and microbial pathogenesis by the regulation of intercellular communications $(19,20)$. In cancer, for instance, exosomes can transfer oncogenic proteins and nucleic acids to modulate the activity of recipient cells, and then shape the microenvironment of tumor (21-23). 
Long non-coding RNA (lncRNA) activated by TGF- $\beta$ (lncRNA-ATB) was initially identified as an lncRNA in hepatocellular carcinoma (HCC). LncRNA-ATB expression was significantly increased hepatocellular carcinoma, which promotes HCC cells invasion and tumor growth. Additionally, lncRNA-ATB can induce epithelial-mesenchymal transition (EMT) and promote invasion by competitively binding and sequestering the microRNA (miRNA or miR)-200 family in HCC (24). Our previous study revealed that lncRNA-ATB promoted the migration and invasion of glioma cells by acting as a competitive endogenous RNA (ceRNA) of miR-200a (25). Recently, plasma lncRNA-ATB expression has been shown to be increased in lung disease, suggesting that circular lncRNA-ATB may play a key role in disease (26).

In this study, we aimed to investigate the functional role of glioma cell-derived exosomal lncRNA-ATB in astrocytes. We describe the effects of glioma cell-derived exosomes on the activation of astrocytes. We demonstrate that lncRNA-ATB in glioma cell-derived exosomes activates astrocytes through the suppression of miR-204-3p and that reactive astrocytes stimulate glioma cell invasion. These results suggest that IncRNA-ATB may prove to be a novel therapeutic target for the treatment of invasive glioma.

\section{Materials and methods}

Cell culture. The A172 and U251 glioma cells were purchased from the Chinese Academy of Sciences (Shanghai, China) and cultured in Dulbecco's modified Eagle's medium (DMEM; HyClone, Logan, UT, USA) with high glucose supplemented with $10 \%$ fetal bovine serum (FBS; Gibco; Thermo Fisher Scientific, Inc., Waltham, MA, USA) and streptomycin $(100 \mu \mathrm{g} / \mathrm{ml})$, penicillin $(100 \mathrm{U} / \mathrm{ml})$. Normal human astrocytes (NHAs) were obtained from Sun Yat-Sen University and cultured in astrocyte medium with low glucose supplemented with $10 \%$ fetal bovine serum. All cell lines were cultured at $37^{\circ} \mathrm{C}$ in a humidified incubator containing $5 \% \mathrm{CO}_{2}$.

Isolation and analysis of exosomes. For exosomes isolation, we first transplanted an equal number of different cells into $10 \mathrm{~cm}$ plates and changed the culture medium with fresh DMEM-supplemented serum, which was depleted of exosomes. The culture medium was collected following centrifugation at $3,000 \mathrm{x} \mathrm{g}$ for $15 \mathrm{~min}$. Exosomes were extracted from the cell culture medium using a Total Exosome Isolation kit (Thermo Fisher Scientific) according to the manufacturer's instructions. The samples were then examined by transmission electron microscopy (TEM) on a JEM 1010 transmission electron microscope at an accelerating voltage of $80 \mathrm{Kv}$. Digital images were obtained using the AMT Imaging System (Advanced Microscopy Techniques Corp., Woburn, MA, USA).

Uptake of exosomes by astrocytes. Exosomes from the A172 and U251 glioma cells were labeled with Dil (Sigma, St. Louis, MO, USA) according to the supplier's instructions, suspended in low serum medium $(10 \mu \mathrm{g} / \mathrm{ml})$, and incubated with the astrocytes for $24 \mathrm{~h}$ at $37^{\circ} \mathrm{C}$. Following incubation, the cells were processed as previously described (27).
miRNA transfection and plasmid construction. miR-204-3p mimic (sequence: GCUGGGAAGGCAAAGGGACGU) and negative control (NC) were designed and synthesized by RiboBio Co., Ltd. (Guangzhou, China). miRNA was transfected into the cells using Lipofectamine 2000 (Invitrogen; Thermo Fisher Scientific, Inc.). The transfection process was conducted according to manufacturer's guidelines.IncRNA ATB sequences (forward,5'-CTCAAGCTTGGC CCTGGGGCTCTGCAA-3' and reverse, 5'-GGAATTCTG GTAAATGAGTCCAAAGTC-3') were synthesized and subcloned into the pCDNA3.1 vector (Sangon Biotech, Shanghai). The pCDNA constructs or the empty vector were transfected into the cells cultured in 6-well plates according to the manufacturer's instructions (Invitrogen; Thermo Fisher Scientific, Inc.).

Immunofluorescence staining. The NHAs were fixed with $4 \%$ paraformaldehyde, and permeabilized with $0.2 \%$ Triton X-100 in $1 \%$ bovine serum albumin (BSA) for $10 \mathrm{~min}$ and blocked with $5 \%$ BSA for $1 \mathrm{~h}$ at room temperature. Incubation with anti-glial fibrillary acidic protein (GFAP) antibodies (1:400, ab7260; Abcam, Cambridge, MA, USA) at $4^{\circ} \mathrm{C}$ overnight was followed by incubation with rabbit IgG, Cy3 (1:100, bs-0295P; Bioss Antibodies Inc., Beijing) at room temperature for $1 \mathrm{~h}$. The cells were mounted with SlowFade Gold antifade reagent with DAPI (Sigma, St. Louis, MO, USA) and images were acquired using fluorescence microscopy (Olympus IX71; Olympus, Tokyo, Japan). Counting the number of lipid droplets containing a specific amount of pixels was carried out using Image J software.

RNA extraction and reverse transcription-quantitative PCR $(R T-q P C R)$. Total RNA was extracted from the cultured cells and exosomes using TRIzol reagent (Invitrogen; Thermo Fisher Scientific, Inc.) according to the manufacturer's instructions. Using a Nanodrop spectrophotometer (Implen $\mathrm{GmbH}$, Munich, Germany), the RNA concentration and quality were determined by the $260 / 280 \mathrm{~nm}$ absorbance. Subsequently, the RNA was reverse transcribed into high capacity cDNA using PrimeScriptTM RT Master Mix (Perfect Real-Time) (Takara Biotechnology, Dalian, China). Maxima SYBR-Green/ROX qPCR Master Mix (Thermo Fisher Scientific, Inc.) was used for quantitative PCR. In brief, each PCR reaction mixture in a total volume of $10 \mu \mathrm{l}$, containing $5 \mu \mathrm{l}$ of $2 \mathrm{X}$ SYBR-Green Master Mix, $1 \mu 1$ of sense and antisense primers, $3 \mu 1$ of cDNA, was run for 45 cycles with denaturation at $95^{\circ} \mathrm{C}$ for $15 \mathrm{sec}$, annealing at $60^{\circ} \mathrm{C}$ for $30 \mathrm{sec}$, and extension at $72^{\circ} \mathrm{C}$ for $30 \mathrm{sec}$. The primers for genes were determined as follows: IncRNA-ATB forward, 5'-ACAAGCTGTGCAGTCTCAGG-3' and reverse, 5'-CTAGGCCCAAAGACAATGGA-3'; GFAP forward, 5'-AGGTCCATGTGGAGCTTGAC-3' and reverse, 5'-GCCATTGCCTCATACTGCGT-3'; and GAPDH forward, 5'-AGCAAGAGCACAAGAGGAAG-3' and reverse, 5'-GGT TGAGCACAGGGTACTTT-3'.

The All-in-One ${ }^{\mathrm{TM}}$ miRNA First-Strand cDNA Synthesis kit (Genecopoeia, Guangzhou, China) was used for miRNA reverse transcription and RT-qPCR was conducted using the All-in-One ${ }^{\mathrm{TM}}$ miRNA qPCR kit (Genecopoeia) for miR-204-3p and U6 (miRQ0022693-1-1/MQP-0202, respectively; RiboBio Co., Ltd.), respectively, using ABI 7100 (Applied Biosystems, 
$\mathbf{A}$

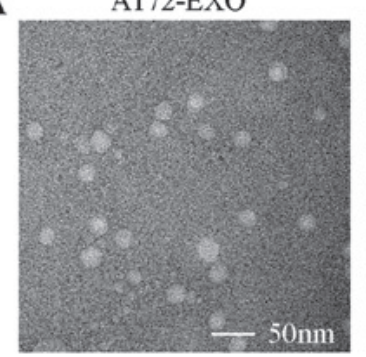

U251-EXO

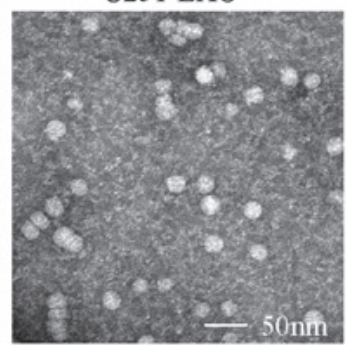

B

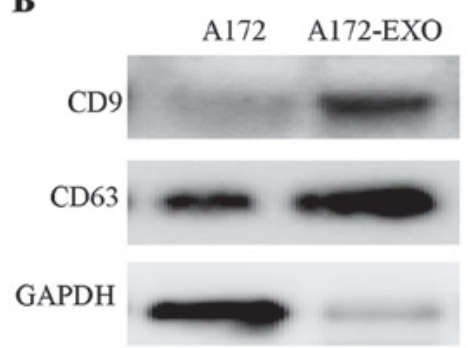

U251 U251-EXO

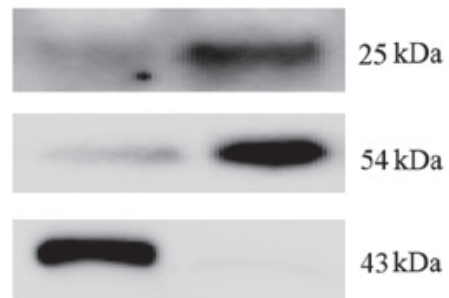

Figure 1. Exosomes released from glioma cells. (A) Transmission electron microscopy for the examination of the morphology and size characterization of exosomes isolated from A172 and U251 glioma cells cultured in conditioned medium. (B) Western blot analysis with anti-CD9 antibody and anti-CD63 antibody, used as markers of exosomes, confirming their correct isolation.

Darmstadt, Germany). For relative quantification, the $2^{-\Delta \Delta \mathrm{Cq}}$ value was calculated and used as an indication of the relative expression levels (28), which was calculated by subtracting the CT values of the control gene from the CT values of lncRNA-ATB, GFAP and miR-204-3p.

Cell migration and invasion assays. The cell migratory and invasive ability was examined using 24-well chambers with an $8 \mu \mathrm{m}$ pore size (Corning, Inc., Corning, NY, USA). A total of $5 \times 10^{3}$ cells were resuspended in $100 \mu \mathrm{l}$ serum-free medium and seeded into the upper chamber with or without pre-coated with $500 \mathrm{ng} / \mathrm{ml}$ Matrigel solution (BD Biosciences, San Jose, CA, USA), while serum-containing medium was placed in the bottom chamber of Transwell plates, following incubation at $37^{\circ} \mathrm{C}$ for $48 \mathrm{~h}$ for the migration and invasion assays. Non-migrated and non-invaded cells from the upper chamber were scraped off using a cotton swab. The migrated and invaded cells on the lower chamber membrane were fixed with $4 \%$ polyoxymethylene and stained with crystal violet (Sigma) at room temperature for $5 \mathrm{~min}$, and dried. Five predetermined fields were counted under a microscope (Olympus IX71, (Olympus; x200 magnification). All assays were performed in triplicate.

Luciferase reporter assays. The 1 ncRNA-ATB fragment containing the predicted miR-204-3p binding site (miRDB, http://mirdb.org/), and the putative sequences of the binding site were cloned into a pmirGlO Dual-luciferase miRNA Target Expression Vector (Promega, Madison, WI, USA) to form the reporter vector pmiRGLO-lncRNA-ATB. PmiRGLO-lncRNA-ATB was co-transfected with miR-204-3p mimics or NC into NHAs using Lipofectamine 2000 (Invitrogen; Thermo Fisher Scientific, Inc.). Following $48 \mathrm{~h}$ of transfection, luciferase assay was carried out using a Dual-Luciferase Reporter Assay System (Promega) according to the manufacturer's instructions. All assays were independently performed in triplicate.

RNA immunoprecipitation. The EZ-Magna RIP RNA-binding protein immunoprecipitation kit (EMD Millipore, Billerica, MA, USA) was used in RNA immunoprecipitation (RIP). RIP was implemented to pull down endogenous miR-204-3p associated with lncRNA-ATB in NHAs, and was performed following the manufacturer's instructions. NHAs were lysed with RIP lysis buffer, and $100 \mu 1$ of cell lysate were incubated with RIP immunoprecipitation buffer containing magnetic beads conjugated with human anti-Argonaute 2 (Ago2) antibody (EMD Millipore) and normal IgG (EMD Millipore) was used as negative control. The samples were incubated with Proteinase $\mathrm{K}$ buffer and then target RNA was extracted using TRIzol reagent (Invitrogen; Thermo Fisher Scientific, Inc.) according to the manufacturer's instructions. Purified RNA was subjected to RT-qPCR analysis.

Western blot analysis. Total proteins were extracted from the cells using RIPA buffer with PMSF (Beyotime Institute of Biotechnology, Shanghai, China) on ice, subjected to $10 \%$ SDS-PAGE gel and electrophoretically transferred onto polyvinylidene difluoride (PVDF) membranes. The membranes were incubated in $5 \%$ non-fat milk dissolved in Tris-buffered saline (TBS) containing $0.1 \%$ Tween-20 for $1.5 \mathrm{~h}$ at room temperature and then incubated with primary antibodies at $4^{\circ} \mathrm{C}$ overnight as follows: CD63, CD9 and GAPDH (1:1,000, ab193349/ab223052/ab9484, respectively; Abcam). Following incubation at room temperature for $2 \mathrm{~h}$ with secondary antibodies (goat anti-rabbit or goat anti-mouse, 1:5,000, ZB-2301/ZB-2305, respectively; ZSGB-BIO, Beijing, China), immune complexes were visualized using the SuperSignal ${ }^{\circledR}$ West Femto Trial kit (Thermo Fisher Scientific, Inc.) and blot bands were scanned using Find-do x6 Tanon (Tanon, Shanghai, China).

Statistical analysis. Experimental data are presented as the means \pm standard deviation (SD). GraphPad Prism V5.0 software (GraphPad Software, Inc., La Jolla, CA, USA) was used for statistical analysis. Differences were analyzed using SPSS 17.0 statistical software with the Student's t-test or one-way ANOVA. A value of $\mathrm{P}<0.05$ was considered to indicate a statistically significant difference.

\section{Results}

Isolation and characterization of glioma cell-derived exosomes. Exosomes purified from the media of the A172 and U251 glioma cells are shown in Fig. 1A, as visualized by TEM. TEM revealed a relatively uniform population of membrane-bound vesicles around $100 \mathrm{~nm}$. Moreover, western blot analyses revealed the isolated particles expressed markers associated with exosomes (CD9 and CD63) (Fig. 1B). Thus, these nanovesicles have the characteristics of exosomes and can be isolated in a consistent manner. 
A

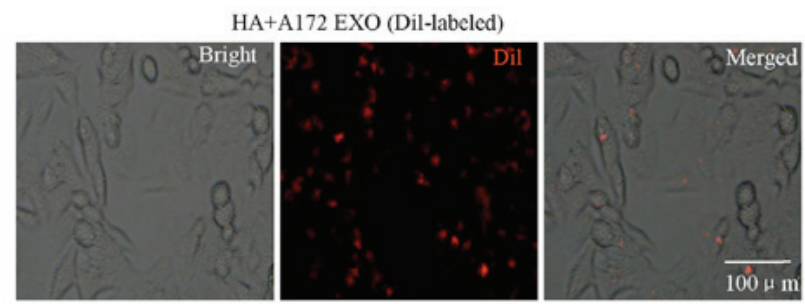

B

HA+U251 EXO (Dil-labeled)
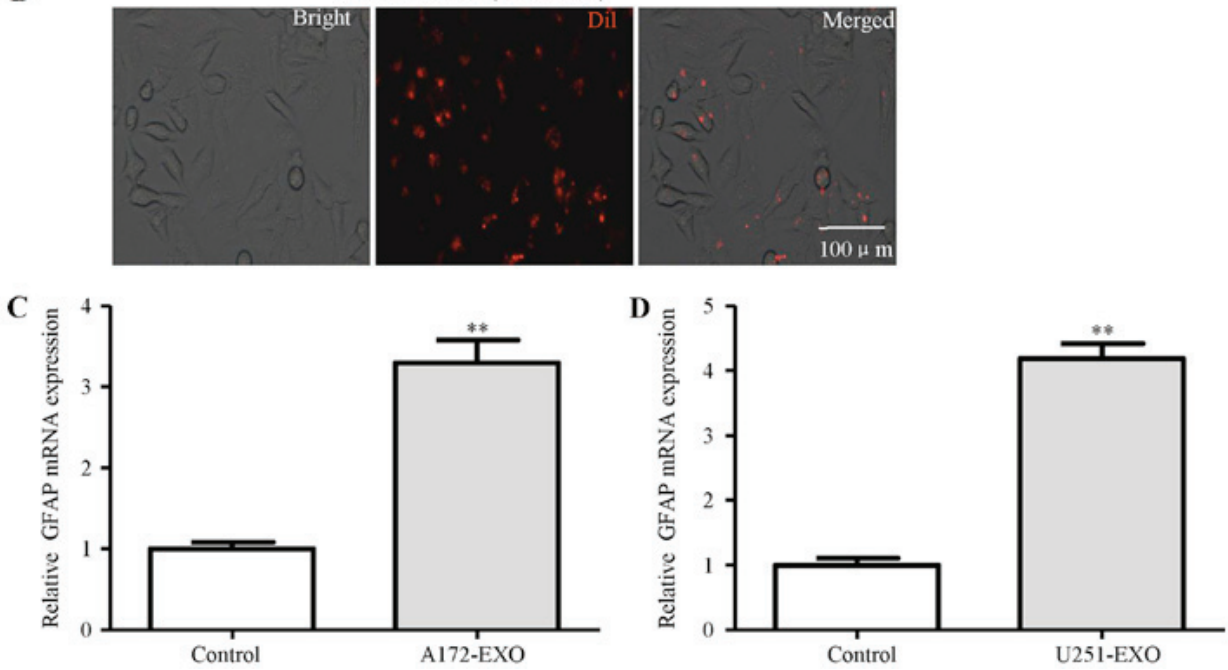

Figure 2. Glioma cell-derived exosomes activate astrocytes. (A and B) Normal human astrocytes (NHAs) were cultured in the absence (control) or presence of glioma cells-derived Dil-labeled exosomes for $24 \mathrm{~h}$. Exosomes were taken up by the NHAs, as shown under a fluorescence fiberscope. NHAs were stained with DAPI (nuclei). (C and D) RT-qPCR analyses of GFAP expression were done on astrocytes $24 \mathrm{~h}$ following treatment with A172 and U251 glioma cell-derived exosomes $(10 \mu \mathrm{g} / \mathrm{ml})$. Untreated cells were used as a control. ${ }^{* *} \mathrm{P}<0.01$ vs. control.

Glioma cell-derived exosomes activate astrocytes. NHAs, a type of normal human brain astrocytes, were selected for use as NHAs in our experimental model. The isolated exosomes from the A172 and U251 glioma cells were labeled with Dil dye (red), washed thoroughly and then added to the NHAs. The uptake of the labeled exosomes by NHAs revealed a red signal under a fluorescence microscope (Fig. 2A and B). These results suggested that the uptake of exosomes by these cells was very efficient. To gain insight into the effects of exosomes derived from the glioma cells on astrocytes, the activation of astrocytes was measured. The results of RT-qPCR revealed that treatment with the A172 and U251 glioma cell-derived exosomes induced the mRNA expression of GFAP, a marker of astrocyte activation (Fig. 2C and D).

IncRNA-ATB in exosomes mediates astrocyte activation. We then examined the mechanisms through which the glioma cells-derived exosomes activate astrocytes. The results of RT-qPCR demonstrated that the exosomes derived from the A172 and U251 glioma cells contained higher levels of IncRNA-ATB when compared with those derived from the parent glioma cells (Fig. 3A and B). To determine whether lncRNA-ATB secreted from the A172 and U251 glioma cells can be transferred to astrocytes via exosomes, we measured the lncRNA-ATB levels in astrocytes treated with exosomes derived from the glioma cells. Similar to that observed for exosome uptake, an increase in the expression of lncRNA-ATB was observed in the NHAs following treatment with the glioma cell-derived exosomes at $24 \mathrm{~h}$ (Fig. 3C and D). To explore the possible functional role of lncRNA-ATB in astrocytes, IncRNA-ATB was transfected into astrocytes. The results of RT-qPCR analysis revealed the elevated expression of lncRNA-ATB in the NHAs (Fig. 4A). The enhanced expression of lncRNA-ATB in the NHAs significantly increased GFAP mRNA expression (Fig. 4B). In addition, the increase in the number of GFAP-positive cells shown by immunofluorescence staining was observed in the NHAs transfected with IncRNA-ATB compared with the empty vector-transfected cells (Fig. 4C). Collectively, these findings indicate that tumor-derived exosomal lncRNA-ATB mediates the activation of astrocytes.

IncRNA-ATB activates astrocytes physically associated with miR-204-3p. Bioinformatics analysis by miRDB revealed putative targeting sites of miR-204-3p and miR-200a shared by 1 cRNA-ATB (Fig. 5A). To further determine whether lncRNA-ATB regulates the expression of miR-204-3p and miR-200a, the NHAs were transfected with lncRNA-ATB or the empty vector. The results revealed that the expression of miR-204-3p and miR-200a was decreased by 69 and $46 \%$ in the IncRNA-ATB vector-transfected cells compared with the empty vector control-transfected cells, respectively (Fig. 5B). Therefore, in the subsequent experiments, we mainly focused on the involvement of miR-204-3p in the lncRNA-ATB-mediated activation of astrocytes.

To validate whether IncRNA-ATB is a target of miR-204-3p in astrocytes, we constructed a luciferase reporter plasmid of lncRNA-ATB containing miR-204-3p binding sites. As shown in Fig. 5C and D, the ectopic expression of miR-204-3p decreased IncRNA-ATB luciferase activity compared with 

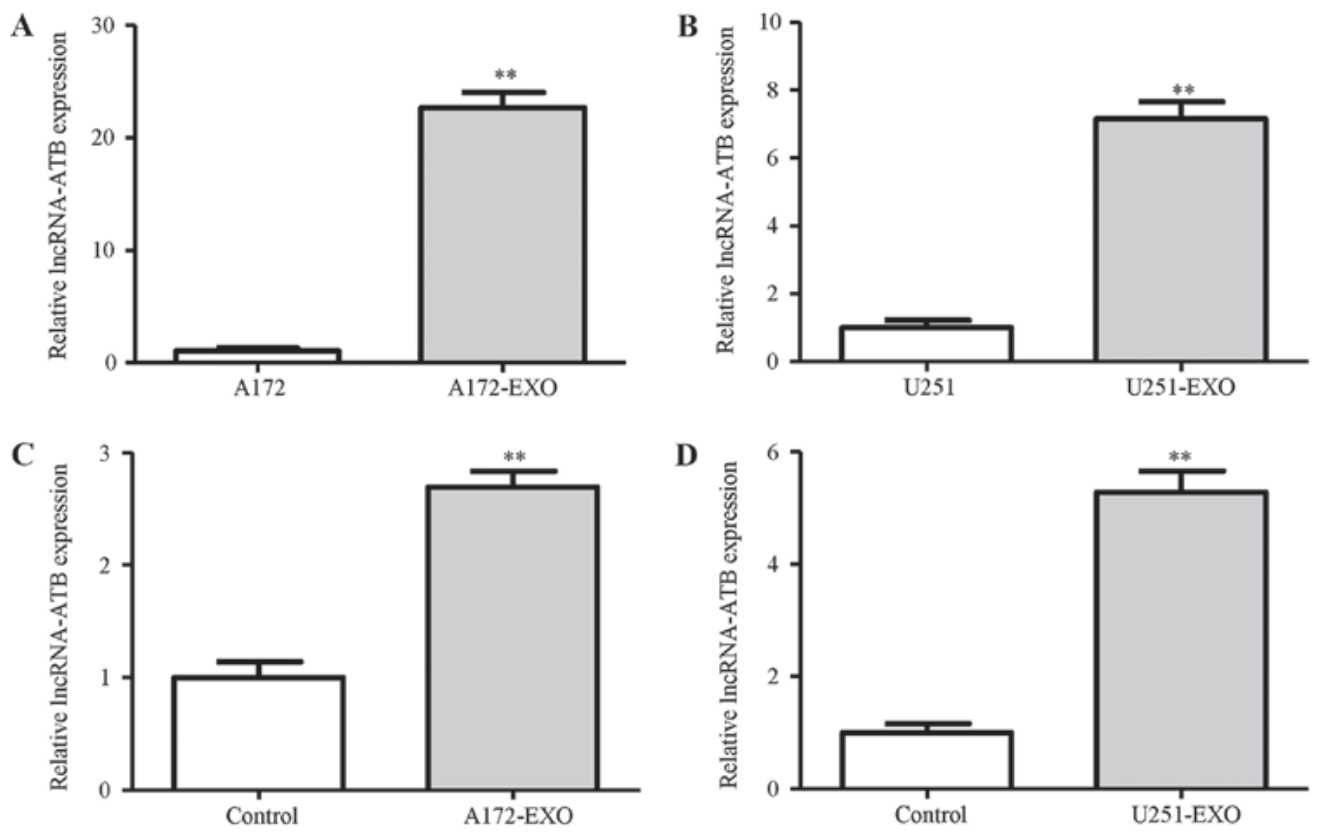

Figure 3. Exosomal lncRNA-ATB is secreted by A172 and U251 glioma cells and mediates astrocyte activation. (A and B) RT-qPCR analysis of exosomes derived from A172 and U251 glioma cells contained higher levels of lncRNA-ATB when compared with those derived from parent glioma cells (control); ${ }^{* *} \mathrm{P}<0.01$ vs. control. (C and D) RT-qPCR analysis of lncRNA-ATB on normal human astrocytes (NHAs) $24 \mathrm{~h}$ following treatment with exosomes derived from A172 and U251 glioma cells. Untreated cells were used as controls. ** $\mathrm{P}<0.01$ vs. control.
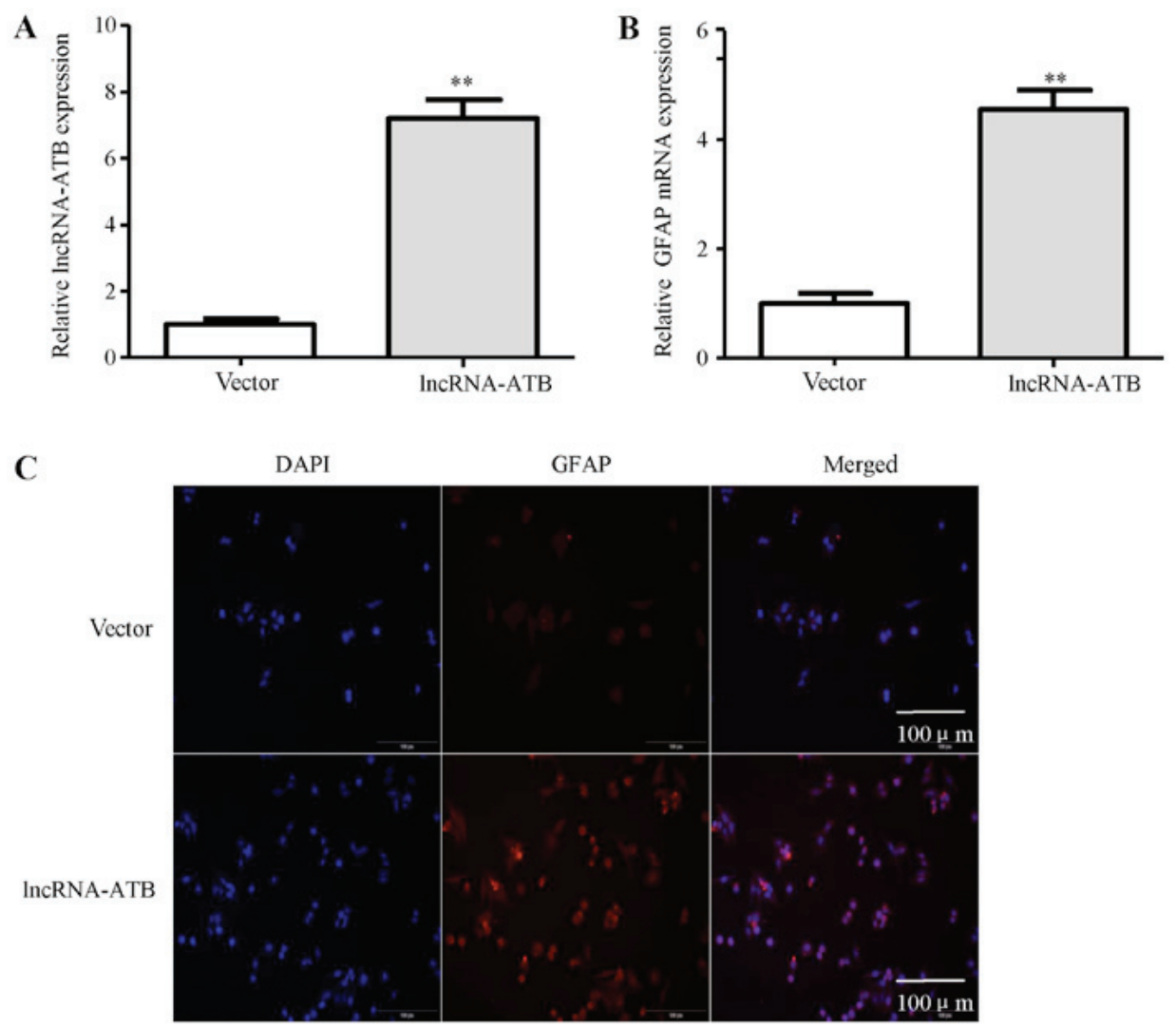

Figure 4. IncRNA-ATB induces astrocyte activation. (A and B) RT-qPCR analysis of lncRNA-ATB and glial fibrillary acidic protein (GFAP) expression in normal human astrocytes (NHAs) transfected with empty vector or lncRNA-ATB plasmid; ${ }^{* *} \mathrm{P}<0.01 \mathrm{vs}$. empty vector. (C) Immunofluorescence staining was performed to assess the protein level of GFAP in NHAs transfected with lncRNA-ATB or empty vector.

the negative control (NC). However, the overexpression of miR-204-3p did not significantly affect lncRNA-ATB expression in the NHAs (Fig. 5E).
miRNAs are known to bind their targets and cause translational repression and/or RNA degradation in an Ago2-dependent manner (25). Thus, in this study, to determine 

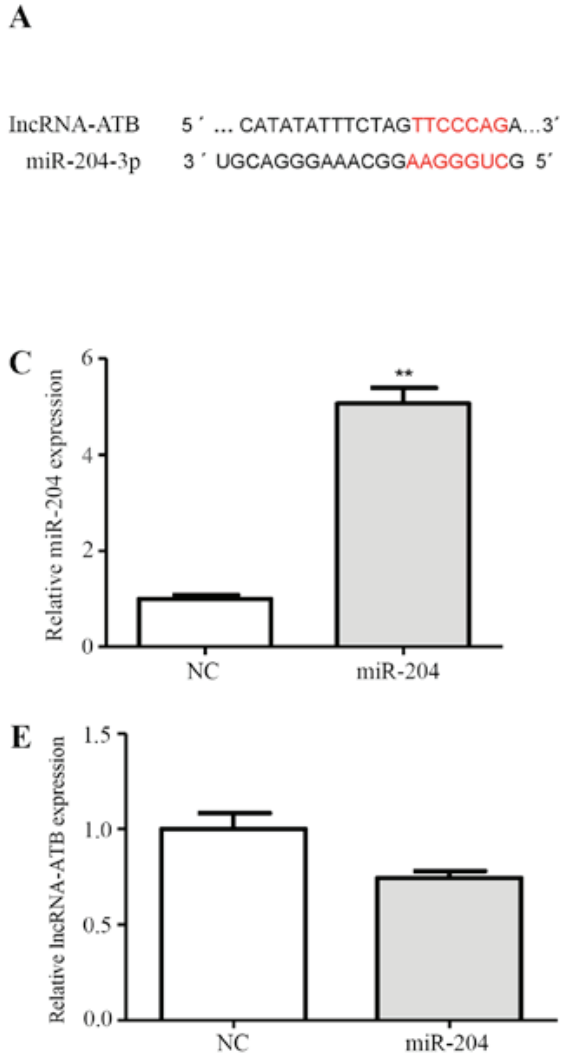
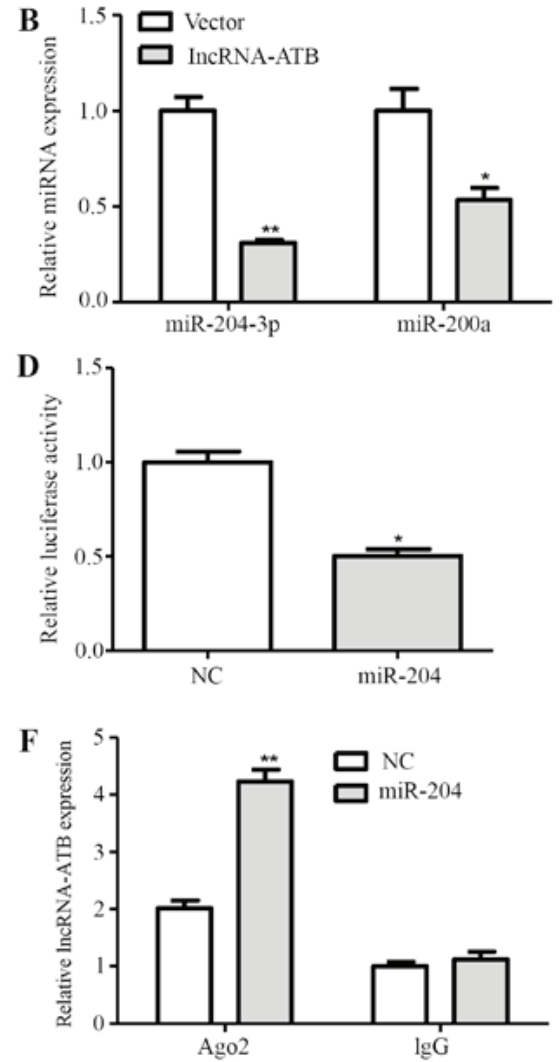

Figure 5. IncRNA-ATB directly targets miR-204-3p in astrocytes. (A) Target gene prediction of miR-204-3p using bioinformatics tools. (B) RT-qPCR assays of miR-204-3p and miR-200a expression in normal human astrocytes (NHAs) transfected with empty vector or lncRNA-ATB plasmid; ${ }^{* *}$ P $<0.01$ vs. vector.

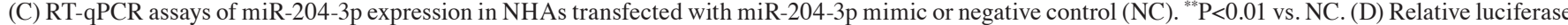
activity of NHAs transfected with miR-204-3p mimic or NC. ${ }^{* *} \mathrm{P}<0.01$ vs. NC. (E) RT-qPCR assays of 1ncRNA-ATB expression in NHAs transfected with miR-204-3p mimic or NC. ${ }^{* *}$ P $<0.01$ vs. NC. (F) RNA-IP with anti-Ago2 antibody was performed in NHAs transfected with miR-204-3p mimic or NC. lncRNA-ATB expression level was detected by RT-qPCR. ${ }^{* *} \mathrm{P}<0.01$ vs. NC.

whether lncRNA-ATB is regulated by miR-204-3p in such a manner, we conducted anti-Ago2 RIP in NHAs transiently overexpressing miR-204-3p. Endogenous lncRNA-ATB pull-down was specifically enriched in the miR-204-3p-transfected NHAs (Fig. 5F), suggesting that miR-204-3p are bona fide lncRNA-ATB-targeting miRNAs. These data demonstrated that miR-204-3p bound to lncRNA-ATB, but did not induce the degradation of 1ncRNA-ATB. All these data suggested that lncRNA-ATB was physically associated with miR-204-3p in the NHAs. In addition, miR-204-3p inhibited GFAP expression in the NHAs compared with the NC (Fig. 6A). As shown in Fig. 6B, the decrease in the number of GFAP-positive cells shown by immunofluorescence staining was observed in the NHAs transfected with miR-204-3p compared with the NC. These results suggest that lncRNA-ATB activates astrocytes partly through the suppression of miR-204-3p.

Astrocytes activated by IncRNA-ATB regulate glioma cell invasion. To determine the effects of astrocytes activated by lncRNA-ATB on glioma cell migration and invasion, we investigated the migration of the A172 and U251 glioma cells co-cultured with vector-transfected NHAs or lncRNA-ATB-transfected NHAs. As a result, the migration of the glioma cells was markedly increased under IncRNA-ATB-NHA culture conditions (Fig. 7A). Similarly, the invasion of the glioma cells was markedly increased
$\mathbf{A}$

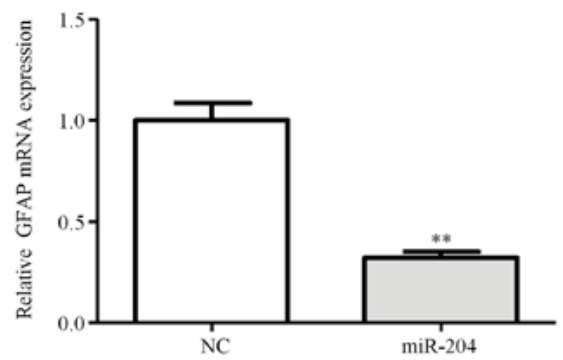

B
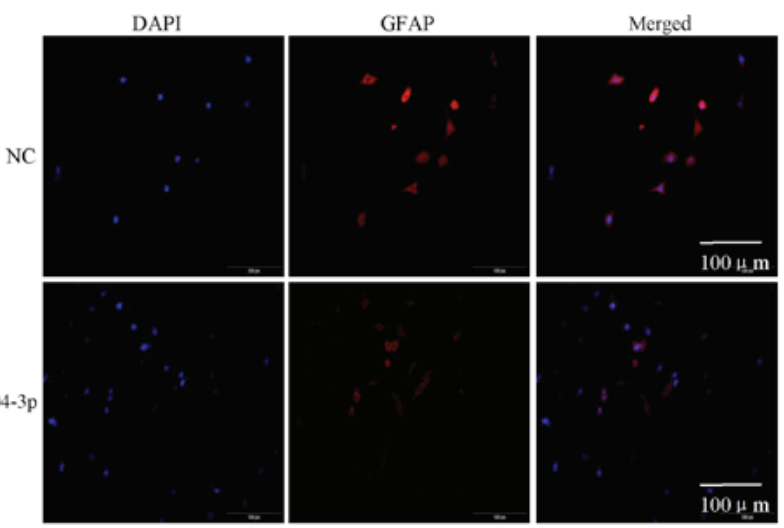

Figure 6. miR-204-3p inhibits astrocyte activation. (A) RT-qPCR assays of glial fibrillary acidic protein (GFAP) expression in normal human astrocytes (NHAs) transfected with miR-204-3p mimic or $\mathrm{NC} ;{ }^{* *} \mathrm{P}<0.01$ vs. negative control (NC). (B) Immunofluorescence staining was performed to assess the protein level of GFAP expression in NHAs transfected with miR-204-3p mimic or NC. 

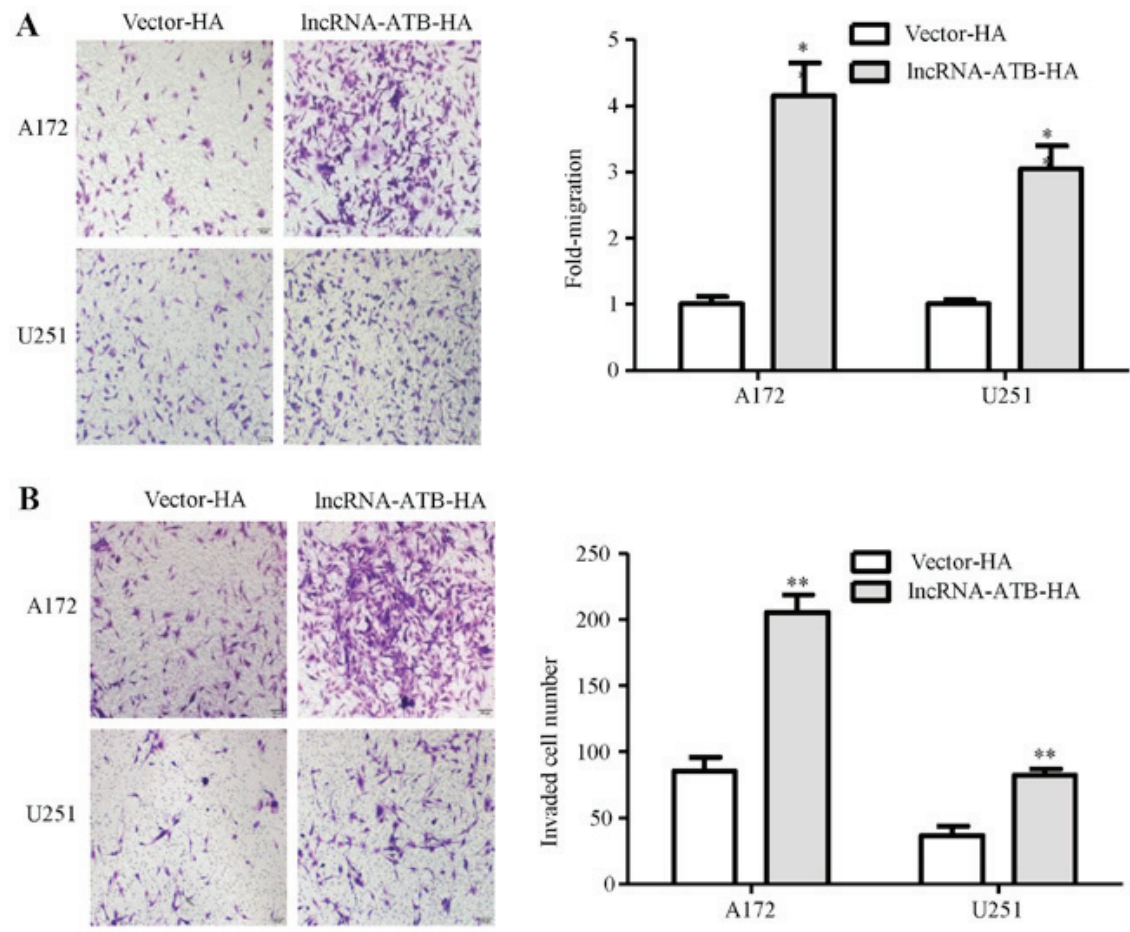

Figure 7. (A) A co-culture Transwell migration assay was performed. A172 and U251 glioma cells in the upper chamber were co-cultured with or without normal human astrocytes (NHAs) in the bottom chamber of the Transwell plate. NHAs in the bottom chamber of the Transwell plate were transfected with empty vector or lncRNA-ATB plasmid; ${ }^{* *} \mathrm{P}<0.01$ vs. vector. (B) A co-culture invasion assay (use Matrigel Transwell chambers) was performed. A172 and U251 glioma cells in the upper chamber were co-cultured with or without NHAs in the bottom chamber of the Transwell plate. NHAs in the bottom chamber of the Transwell plate were transfected with vector or lncRNA-ATB plasmid; ${ }^{* *} \mathrm{P}<0.01$ vs. vector.

when the cells were cultured with lncRNA-ATB-NHAs in the bottom chamber (Fig. 7B).

\section{Discussion}

Gliomas are known to alter the phenotype of normal cells in their environs to promote glioma invasion, and astrocytes are key players in this process. A number of interactions between gliomas and astrocytes are regulated through chemokines and cytokines in the secretome (29). This study supports an additional form of communication in which lncRNAs are transferred from glioma cells into astrocytes via exosomes. The transfer of lncRNA-ATB in glioma cell-derived exosomes resulted in elevated levels in astrocytes and in the suppression of miR-204-3p. Although other miRNAs and proteins exist in these exsomes, the effects may be combinatorial.

Exosome transport is believed to be an effective means for modulating cell signaling and biological function in recipient cells (30). Several studies have described the role of exosomes in the tumor immune response, tumor invasiveness and metastasis (31-33). Importantly, the ability of exosomes shed by tumor cells to transfer the malignant phenotype to normal cells is mediated through the delivery of nucleic acids and proteins, suggesting an important mechanism for 'dissemination' of the tumor (34). Recently, GBM-derived exosomes have been shown to promote angiogenesis during hypoxia, by the transferring of mRNA transcripts and proteins to vascular endothelial cells and pericytes $(35,36)$. However, the contribution of exosomes to the regulation of the biological function of astrocytes is poorly understood. In the present study, our data revealed that exosomes derived from glioma cells were taken up by astrocytes. Moreover, the exosomes exerted a promoting effect on the activation of astrocytes, supporting the notion that exosomes released from malignant cells can affect the functions of surrounding cells. A better characterization of glioma cell-derived exosome contents and the mechanisms of astrocyte interactions are necessary to reverse exosome-induced dysfunction.

Increasing evidence indicates that tumor-derived exosomes can regulate the functions of recipient cells probably through delivering their carried non-coding RNAs. For example, high levels of miR-451/miR-21 in GBM-EVs have been shown to be delivered to microglia, presumably as a means for the tumor to manipulate its environs (29). Qu et al reported that lncARSR could be incorporated into exosomes and transmitted to sensitive cells, thus disseminating sunitinib resistance in renal cancer (37). In this study, our results suggested that lncRNA-ATB embedded in exosomes derived from glioma cells conferred the activation phenotype to recipient astrocytes. Recently, plasma lncRNA-ATB expression has been shown to be increased in lung disease (26). These findings suggest that circular lncRNA-ATB may play a central role in glioma.

Accumulating evidences indicates the common existence of a widespread interaction network of ceRNAs. IncRNAs may carry out its functions by targeting miRNAs and regulating their functional roles. IncRNA-ATB promotes tumor cell invasion and plays a key role in the distant metastasis of HCC by negatively regulating the miR-200 family (24). Our previous study revealed that IncRNA-ATB was abnormally upregulated in glioma, and patients with glioma with a high lncRNA-ATB expression had a shorter overall survival time. IncRNA-ATB functions as a ceRNA by decreasing miR-200a expression, and 
upregulating TGF- $\beta 2$ expression in human glioma cells (25). In this study, we demonstrated that lncRNA-ATB overexpression in astrocytes significantly decreased miR-204-3p, and slightly (although still significantly) decreased miR-200a expression, compared with the empty vector-transfected cells. These results indicate that the regulatory mechanism of lncRNA-ATB may be cell-specific. The results of RNA-IP assay revealed that the expression of IncRNA-ATB immunoprecipitated with in the miR-204-3p overexpression was markedly increased. In addition, miR-204-3p inhibited astrocyte activation. Therefore, these results suggest that lncRNA-ATB may carry out its functions by the suppression of miR-204-3p in astrocytes. Further studies are warranted in order to fully elucidate the molecular mechanisms through which miR-204-3p inhibits astrocyte activation.

Astrocytes are the most abundant cell type in the brain and regulate the homeostasis of the brain microenvironment (38). Astrocytes represent a reactive phenotype when they contact tumor cells, expressing high levels of GFAP (39). There is evidence to indicate that reactive astrocytes promote the brain metastasis of lung and breast cancer cells by secreting various cytokines $(40,41)$. Reactive astrocytes assist the parenchymal infiltrative ability of glioma cells and further augment glioma malignancy $(42,43)$. In this study, we demonstrated that astrocytes activated by lncRNA-ATB promoted the migration and invasion of glioma cells. Reactive astrocytes secrete chemokines or cytokines, such as TGF- $\beta$, among others, to promote tumor cell migration and invasion (44). Recently, studies have reported that IncRNA-ATB governs the autocrine secretion of TGF- $\beta$ and increases the expression of by TGF- $\beta$ via miR-200s $(45,46)$. This evidence suggests that exosomal lncRNA-ATB may activate astrocytes to secrete TGF- $\beta$, which promotes the migration and invasion of glioma cells. However, the mechanisms through which NHAs activated by lncRNA-ATB plays its role in the regulation of glioma cell migration/invasion is not yet clear, which is the field we will focus on in subsequent study.

In conclusion, our results indicate that glioma cell-derived exosomal lncRNA-ATB change phenotype of astrocytes. In addition, exosomal lncRNA-ATB targeted and repressed of miR-204-3p in astrocytes, which in turn promotes the invasion of glioma cells. The findings of this study provide a novel molecular mechanism underlying the crosstalk between glioma cells and astrocytes to promote the invasion of glioma, which contributes to efficient therapeutic strategies for the treatment of invasive glioma.

\section{Acknowledgements}

Not applicable.

\section{Funding}

This study was supported by the National Natural Science Foundation of China (nos. 81402078 and 81502149), the Natural Science Foundation of Anhui Province (nos. 1608085MH225 and 1508085MH194), Key Research and Development Plan Project of Anhui Province (no. 1804h08020270), the Academic Funding Project for Top Talents in Colleges and Universities in Anhui Province (no. gxbjZD10), and the Nova Pew Plan of the Second Affiliated Hospital of Anhui Medical University (no. 2017KA01).

\section{Availability of data and materials}

All data generated or analyzed during this study are included in this published article.

\section{Authors' contributions}

EBB and EFC conceived and designed the experiments. EBB, YDX, ZHY and FT, CCM performed the experiments and drafted the manuscript. HLW and BZ contributed to the design of this study and helped to draft the manuscript. BZ supervised the whole study and revised the manuscript. All authors have read and approved the final manuscript.

\section{Ethics approval and consent to participate}

Not applicable.

\section{Patient consent for publication}

Not applicable.

\section{Competing interests}

The authors declare that they have no competing interests.

\section{References}

1. Johnson DR and O'Neill BP: Glioblastoma survival in the United States before and during the temozolomide era. J Neurooncol 107: 359-364, 2012.

2. Avril T, Vauleon E, Tanguy-Royer S, Mosser J and Quillien V: Mechanisms of immunomodulation in human glioblastoma. Immunotherapy 3 (Suppl 4): 42-44, 2011.

3. Coniglio SJ and Segall JE: Review: Molecular mechanism of microglia stimulated glioblastoma invasion. Matrix Biol 32: 372-380, 2013.

4. D'Asti E, Garnier D, Lee TH, Montermini L, Meehan B and Rak J: Oncogenic extracellular vesicles in brain tumor progression. Front Physiol 3: 294, 2012.

5. Fidler IJ, Balasubramanian K, Lin Q, Kim SW and Kim SJ: The brain microenvironment and cancer metastasis. Mol Cells 30: 93-98, 2010.

6. Katz AM, Amankulor NM, Pitter K, Helmy K, Squatrito M and Holland EC: Astrocyte-specific expression patterns associated with the PDGF-induced glioma microenvironment. PLoS One 7: e32453, 2012.

7. Lee J, Borboa AK, Baird A and Eliceiri BP: Non-invasive quantification of brain tumor-induced astrogliosis. BMC Neurosci 12: 9, 2011.

8. Tang MK and Wong AS: Exosomes: Emerging biomarkers and targets for ovarian cancer. Cancer Lett 367: 26-33, 2015.

9. Théry C: Exosomes: Secreted vesicles and intercellular communications. F1000 Biol Rep 3: 15, 2011.

10. Batrakova EV and Kim MS: Using exosomes, naturally-equipped nanocarriers, for drug delivery. J Control Release 219: 396-405, 2015.

11. Aalberts M, van Dissel-Emiliani FM, van Adrichem NP, van Wijnen M, Wauben MH, Stout TA and Stoorvogel W: Identification of distinct populations of prostasomes that differentially express prostate stem cell antigen, annexin A1, and GLIPR2 in humans. Biol Reprod 86: 82, 2012.

12. Friand V, David G and Zimmermann P: Syntenin and syndecan in the biogenesis of exosomes. Biol Cell 107: 331-341, 2015.

13. Hagiwara K, Ochiya T and Kosaka N: A paradigm shift for extracellular vesicles as small RNA carriers: From cellular waste elimination to therapeutic applications. Drug Deliv Transl Res 4: 31-37, 2014

14. Khalyfa A and Gozal D: Exosomal miRNAs as potential biomarkers of cardiovascular risk in children. J Transl Med 12: 162,2014 
15. Madison MN and Okeoma CM: Exosomes: Implications in HIV-1 Pathogenesis. Viruses 7: 4093-4118, 2015.

16. Hirsch E, Hilfiker-Kleiner D, Balligand JL, Tarone G, De Windt L, Bauersachs J, Ferdinandy P, Davidson S, Hausenloy DJ and Schulz R: Interaction of the heart and its close and distant neighbours: Report of the Meeting of the ESC Working Groups Myocardial Function and Cellular Biology. Cardiovasc Res 99: 595-599, 2013.

17. Lugini L, Cecchetti S, Huber V, Luciani F, Macchia G, Spadaro F, Paris L, Abalsamo L, Colone M, Molinari A, et al: Immune surveillance properties of human NK cell-derived exosomes. J Immunol 189: 2833-2842, 2012.

18. Zhu $\mathrm{H}$ and Fan GC: Extracellular/circulating microRNAs and their potential role in cardiovascular disease. Am J Cardiovasc Dis 1: 138-149, 2011.

19. Madison MN, Jones PH and Okeoma CM: Exosomes in human semen restrict HIV-1 transmission by vaginal cells and block intravaginal replication of LP-BM5 murine AIDS virus complex. Virology 482: 189-201, 2015.

20. Vojtech L, Woo S, Hughes S, Levy C, Ballweber L, Sauteraud RP, Strobl J, Westerberg K, Gottardo R, Tewari M, et al: Exosomes in human semen carry a distinctive repertoire of small non-coding RNAs with potential regulatory functions. Nucleic Acids Res 42: 7290-7304, 2014.

21. Clayton A: Cancer cells use exosomes as tools to manipulate immunity and the microenvironment. OncoImmunology 1: 78-80, 2012.

22. Epple LM, Griffiths SG, Dechkovskaia AM, Dusto NL, White J, Ouellette RJ, Anchordoquy TJ, Bemis LT and Graner MW: Medulloblastoma exosome proteomics yield functional roles for extracellular vesicles. PLoS One 7: e42064, 2012.

23. Taylor DD and Gercel-Taylor C: Exosomes/microvesicles: Mediators of cancer-associated immunosuppressive microenvironments. Semin Immunopathol 33: 441-454, 2011.

24. Yuan JH, Yang F, Wang F, Ma JZ, Guo YJ, Tao QF, Liu F, Pan W, Wang TT, Zhou CC, et al: A long noncoding RNA activated by TGF- $\beta$ promotes the invasion-metastasis cascade in hepatocellular carcinoma. Cancer Cell 25: 666-681, 2014.

25. Ma CC, Xiong Z, Zhu GN, Wang C, Zong G, Wang HL, Bian EB and Zhao B: Long non-coding RNA ATB promotes glioma malignancy by negatively regulating miR-200a. J Exp Clin Cancer Res 35: 90, 2016.

26. Ma J, Cui X, Rong Y, Zhou Y, Guo Y, Zhou M, Xiao L and Chen W: Plasma LncRNA-ATB, a Potential Biomarker for Diagnosis of Patients with Coal Workers' Pneumoconiosis: A Case-Control Study. Int J Mol Sci 17: 17, 2016.

27. Zhou W, Fong MY, Min Y, Somlo G, Liu L, Palomares MR, Yu Y Chow A, O'Connor ST, Chin AR, et al: Cancer-secreted miR-105 destroys vascular endothelial barriers to promote metastasis. Cancer Cell 25: 501-515, 2014

28. Livak KJ and Schmittgen TD: Analysis of relative gene expression data using real-time quantitative PCR and the 2(-Delta Delta C(T)) method. Methods 25: 402-408, 2001.

29. van der Vos KE, Abels ER, Zhang X, Lai C, Carrizosa E, Oakley D, Prabhakar S, Mardini O, Crommentuijn MH, Skog J, et al: Directly visualized glioblastoma-derived extracellular vesicles transfer RNA to microglia/macrophages in the brain. Neuro-oncol 18: 58-69, 2016.

30. Melo SA, Sugimoto H, O'Connell JT, Kato N, Villanueva A, Vidal A, Qiu L, Vitkin E, Perelman LT, Melo CA, et al: Cancer exosomes perform cell-independent microRNA biogenesis and promote tumorigenesis. Cancer Cell 26: 707-721, 2014.

31. Clayton A, Mitchell JP, Court J, Mason MD and Tabi Z: Human tumor-derived exosomes selectively impair lymphocyte responses to interleukin-2. Cancer Res 67: 7458-7466, 2007.
32. Janowska-Wieczorek A, Wysoczynski M, Kijowski J, Marquez-Curtis L, Machalinski B, Ratajczak J and Ratajczak MZ: Microvesicles derived from activated platelets induce metastasis and angiogenesis in lung cancer. Int J Cancer 113: 752-760, 2005.

33. EL Andaloussi S, Mäger I, Breakefield XO and Wood MJ Extracellular vesicles: Biology and emerging therapeutic opportunities. Nat Rev Drug Discov 12: 347-357, 2013.

34. Milane L, Singh A, Mattheolabakis G, Suresh M and Amiji MM: Exosome mediated communication within the tumor microenvironment. J Control Release 219: 278-294, 2015.

35. Kucharzewska P, Christianson HC, Welch JE, Svensson KJ, Fredlund E, Ringnér M, Mörgelin M, Bourseau-Guilmain E, Bengzon J and Belting M: Exosomes reflect the hypoxic status of glioma cells and mediate hypoxia-dependent activation of vascular cells during tumor development. Proc Natl Acad Sci USA 110: 7312-7317, 2013.

36. Svensson KJ, Kucharzewska P, Christianson HC, Sköld S, Löfstedt T, Johansson MC, Mörgelin M, Bengzon J, Ruf W and Belting M: Hypoxia triggers a proangiogenic pathway involving cancer cell microvesicles and PAR-2-mediated heparin-binding EGF signaling in endothelial cells. Proc Natl Acad Sci USA 108: 13147-13152, 2011.

37. Qu L, Ding J, Chen C, Wu ZJ, Liu B, Gao Y, Chen W, Liu F, Sun W, Li XF, et al: Exosome-Transmitted lncARSR Promotes Sunitinib Resistance in Renal Cancer by Acting as a Competing Endogenous RNA. Cancer Cell 29: 653-668, 2016.

38. Grosche J, Matyash V, Möller T, Verkhratsky A, Reichenbach A and Kettenmann $\mathrm{H}$ : Microdomains for neuron-glia interaction: Parallel fiber signaling to Bergmann glial cells. Nat Neurosci 2: 139-143, 1999.

39. Gagliano N, Costa F, Cossetti C, Pettinari L, Bassi R, Chiriva-Internati $M$, Cobos $E$, Gioia $M$ and Pluchino $S$ : Glioma-astrocyte interaction modifies the astrocyte phenotype in a co-culture experimental model. Oncol Rep 22: 1349-1356, 2009.

40. Fitzgerald DP, Palmieri D, Hua E, Hargrave E, Herring JM, Qian Y, Vega-Valle E, Weil RJ, Stark AM, Vortmeyer AO, et al: Reactive glia are recruited by highly proliferative brain metastases of breast cancer and promote tumor cell colonization. Clin Exp Metastasis 25: 799-810, 2008.

41. Seike T, Fujita K, Yamakawa Y, Kido MA, Takiguchi S, Teramoto N, Iguchi $\mathrm{H}$ and Noda $\mathrm{M}$ : Interaction between lung cancer cells and astrocytes via specific inflammatory cytokines in the microenvironment of brain metastasis. Clin Exp Metastasis 28: 13-25, 2011.

42. Barbero S, Bajetto A, Bonavia R, Porcile C, Piccioli P, Pirani P, Ravetti JL, Zona G, Spaziante R, Florio T, et al: Expression of the chemokine receptor CXCR4 and its ligand stromal cell-derived factor 1 in human brain tumors and their involvement in glial proliferation in vitro. Ann N Y Acad Sci 973: 60-69, 2002.

43. Biasoli D, Sobrinho MF, da Fonseca AC, de Matos DG, Romão L, de Moraes Maciel R, Rehen SK, Moura-Neto V, Borges HL and Lima FR: Glioblastoma cells inhibit astrocytic p53-expression favoring cancer malignancy. Oncogenesis 3: e123, 2014

44. Chen W, Xia T, Wang D, Huang B, Zhao P, Wang J, Qu X and Li X: Human astrocytes secrete IL-6 to promote glioma migration and invasion through upregulation of cytomembrane MMP14. Oncotarget 7: 62425-62438, 2016.

45. Lei K, Liang X, Gao Y, Xu B, Xu Y, Li Y, Tao Y, Shi W and Liu J: Lnc-ATB contributes to gastric cancer growth through a MiR-141-3p/TGF 32 feedback loop. Biochem Biophys Res Commun 484: 514-521, 2017.

46. Zhu HY, Bai WD, Li C, Zheng Z, Guan H, Liu JQ, Yang XK, Han SC, Gao JX, Wang HT, et al: Knockdown of lncRNA-ATB suppresses autocrine secretion of TGF- $\beta 2$ by targeting ZNF217 via miR-200c in keloid fibroblasts. Sci Rep 6: 24728, 2016. 